\title{
Inflation from Charged Scalar and Primordial Magnetic Fields?
}

\author{
Razieh Emami \\ Department of Physics, Shahid Beheshti University, G.C., Evin, Tehran 19839, Iran \\ Hassan Firouzjahi* \\ School of Physics, Institute for Research in Fundamental Sciences (IPM), \\ P. O. Box 19395-5531, Tehran, Iran \\ M. Sadegh Movahed \\ Department of Physics, Shahid Beheshti University, \\ G.C., Evin, Tehran 19839, Iran, and \\ School of Astronomy, Institute for Research in Fundamental Sciences (IPM), \\ P. O. Box 19395-5531, Tehran, Iran
}

\begin{abstract}
A model of inflation is presented where the inflaton field is a complex scalar field coupled to a $U(1)$ gauge field. Due to the axial symmetry of the potential, the inflation is driven by the radial direction while the angular field is gauged by $U(1)$. Due to the coupling of the inflaton to the gauge field, a time dependent mass term for the gauge field is generated dynamically and conformal invariance is broken. We study whether a significant amount of primordial magnetic fields can be generated during inflation by allowing a time-dependent $U(1)$ gauge kinetic coupling.

Keywords : Inflation, Primordial Magnetic Fields

PACS numbers:
\end{abstract}

*Electronic address: firouz@ipm.ir

${ }^{\dagger}$ Electronic address: m.s.movahed@ipm.ir 


\section{INTRODUCTION}

Astronomical observations indicate the existence of magnetic fields in galaxies and also in cosmological scales with coherence lengths as big as $1 \mathrm{Mpc}$ and with the magnitude of order $10^{-7}$ Gauss, for more details see [1 13$]$. The origin of the cosmic magnetic fields are not well-understood. One possible explanation is that they may have primordial origin which are later amplified by galactic dynamo mechanism into the current observed value. The estimation for the magnitude of the primordial seed is not certain. Conventionally, it is assumed that a seed in the range $10^{-25}-10^{-15}$ Gauss at the time of matter-radiation decoupling may be required for dynamo mechanism to produce the current observational value. This lower bound may be relaxed to $10^{-30}$ Gauss in a flat dark energy dominated Universe [4].

Starting with the work of Turner and Widrow [5], during past two decades there were some attempts to obtain primordial magnetic seed through inflation [6 43]. In particular, inflation can naturally stretch small micro-physical scales inside a casual patch into the cosmological sizes. This can provide an elegant mechanism to explain the coherence of magnetic fields on distances much bigger than astrophysical scales. The key obstacle, however, in producing magnetic field during inflation (in general in a Friedmann-Robertson-Walker (FRW) background) is the conformal invariance. To produce magnetic field one has to break the conformal invariance of the classical electrodynamics. There are several mechanisms to break conformal invariance. These include: coupling electrodynamics non-minimally to gravity which produce a time-dependent mass for photon [5, 16], introducing a time-dependent gauge kinetic coupling [6 -9, 18-29, 43] , and coupling the photon to a charged scalar field $[5,10-12,14,16]$.

In this paper we combine the last two mentioned methods together in order to break the conformal invariance and obtain an appreciable amount of seed magnetic field. We consider the model where the inflaton field is a complex scalar field coupled to a $U(1)$ gauge field. The conformal invariance is broken dynamically when the scalar field is displaced from its minimum during inflation. Furthermore, the time-dependence of the gauge kinetic coupling as in [22, 43] can help to produce a considerable amount of primordial magnetic fields seed required for galactic dynamo. As indicated recently in [43], the key constraints in models with time-varying gauge coupling are (a): the requirement that the time-dependent gauge 
coupling remains small for all time during inflation, so the perturbative gauge theory is under control and (b): one must check that the strong back reaction from gauge field does not destroy the inflationary background. We shall see that these constraints in addition with the requirement to have sufficient inflation basically control the amplitude of the magnetic fields seed produced.

This paper is organized as follows. In section [I we present our set up. In section III the magnetic fields produced in our model are studied followed by discussions and conclusion in section [V]. While this paper was in its final stage, the work [44] appeared which has some overlaps with our studies.

\section{SET UP}

Here we present our set up. It contains a complex scalar field, $\phi$, coupled to a $U(1)$ gauge field, $A_{\mu}$, with a time dependent gauge kinetic coupling, $I(t)$. The action is

$$
S=\int d^{4} x \sqrt{-g}\left[\frac{M_{P}^{2}}{2} R-\frac{1}{2} D_{\mu} \phi D^{\mu} \phi^{\dagger}-\frac{I^{2}(t)}{4} F_{\mu \nu} F^{\mu \nu}-V\left(\phi, \phi^{\dagger}\right)\right],
$$

where $M_{P}^{-2}=8 \pi G$ and $G$ being the Newton constant. The covariant derivative is given by

$$
D_{\mu} \phi=\partial_{\mu} \phi+i e \phi A_{\mu}
$$

where $e$ is the dimensionless gauge coupling of $A_{\mu}$ to $\phi$. As usual, the gauge field strength is given by

$$
F_{\mu \nu}=\partial_{\mu} A_{\nu}-\partial_{\nu} A_{\mu}
$$

Motivated by [22, 43] we have entertained the possibility that the gauge kinetic coupling is time dependent. This may arise for example in string theory setup where the gauge kinetic action is coupled to the dilaton which is running with time. As suggested in [43] one may also consider that the time dependence of $I(t)$ originates from the time dependence of $e(t)$. This may be obtained by replacing $A_{\mu} \rightarrow A_{\mu} / e(t)$. But this has the problem that the kinetic action $F_{\mu \nu} F^{\mu \nu}$ becomes modified under $A_{\mu} \rightarrow A_{\mu} / e(t)$ and the gauge invariant is lost. At the phenomenological level, we shall take $e$ to be constant and assume the time dependence of $I(t)$ originates from the coupling of the gauge kinetic action to other fields such as dilaton. However, the dynamics of the dilaton should be such that it does not destroy the slow-roll 
properties of the inflationary potential. This may be a non-trivial task, but we shall take the phenomenological view of [22, 43] and proceed with action represented in Eq. (11).

We work with potentials which have axial symmetry where $V$ is only a function of $\phi \phi^{\dagger}=$ $|\phi|^{2}$. It is more instructive to decompose the inflaton field into the radial and angular parts

$$
\phi(x)=\rho(x) e^{i \theta(x)},
$$

so $V=V(\rho)$. As usual, the action, Eq. (11), is invariant under local gauge transformation

$$
\begin{aligned}
A_{\mu} & \rightarrow A_{\mu}-\frac{1}{e} \partial_{\mu} \epsilon(x) \\
\theta & \rightarrow \theta+\epsilon(x) .
\end{aligned}
$$

With this decomposition, Eq. (11) is transformed into:

$S=\int d^{4} x \sqrt{-g}\left[\frac{M_{P}^{2}}{2} R-\frac{1}{2} \partial_{\mu} \rho \partial^{\mu} \rho-\frac{\rho^{2}}{2}\left(\partial_{\mu} \theta+e A_{\mu}\right)\left(\partial^{\mu} \theta+e A^{\mu}\right)-\frac{I(t)^{2}}{4} F_{\mu \nu} F^{\mu \nu}-V(\rho)\right](6)$ The corresponding Klein-Gordon equations of motion are:

$$
\begin{aligned}
& 0=\partial_{\mu} J^{\mu} \\
& 0=\partial_{\mu}\left[\sqrt{-g} \partial^{\mu} \rho\right]-\frac{J_{\mu} J^{\mu}}{\rho^{3} \sqrt{-g}}-\sqrt{-g} V_{\rho},
\end{aligned}
$$

accompanied by with the Maxwell's equation

$$
\partial_{\mu}\left(\sqrt{-g} I(t)^{2} F^{\mu \nu}\right)=e J^{\nu}
$$

where the current, $J^{\nu}$, is defined by

$$
J^{\nu} \equiv \rho^{2} \sqrt{-g}\left(\partial^{\nu} \theta+e A^{\nu}\right) .
$$

The conservation of $J^{\mu}$ from Eq. (7) is a manifestation of the axial symmetry imposed on $V$. Interestingly, Eq. (7) is not independent from Maxwell's equation, where $F^{\mu \nu}$ being anti-symmetic leads to $\partial_{\mu} \partial_{\nu} F^{\mu \nu}=\partial_{\mu} J^{\mu}=0$.

Finally, the stress energy momentum tensor, $T_{\alpha \beta}$, for the Einstein equation, $G_{\alpha \beta}=$ $8 \pi G T_{\alpha \beta}$, is:

$T_{\alpha \beta}=\frac{-I(t)^{2}}{4} g_{\alpha \beta} F_{\mu \nu} F^{\mu \nu}+I(t)^{2} F_{\alpha \mu} F_{\beta}^{\mu}+\partial_{\alpha} \rho \partial_{\beta} \rho+\frac{J_{\alpha} J_{\beta}}{\rho^{2}|g|}-g_{\alpha \beta}\left[\frac{1}{2} \partial_{\mu} \rho \partial^{\mu} \rho+\frac{J_{\mu} J^{\mu}}{2 \rho^{2}|g|}+V\right](11)$

At the background level, we start with the isotropic and homogeneous FRW space-time with the metric

$$
d s^{2}=-d t^{2}+a(t)^{2} d \overrightarrow{\mathbf{x}}^{2} .
$$


Considering the $\nu=0$ component of the Maxwell equation (91) implies that

$$
J^{0}=\rho^{2} a^{3}\left(\dot{\theta}+e A^{0}\right)=0 .
$$

This indicates that the total electric charge is zero [45]. This is somewhat similar to usual angular momentum conservation where in the absence of gauge field, an axial symmetric potential leads to angular momentum conservation $\partial_{t}\left(a^{3} \rho^{2} \dot{\theta}\right)=0$.

The gauge field $A_{\mu}$ is invariant under gauge transformation Eq. (5). We need to fix the gauge to study the physical independent degrees of freedom. We choose the Coloumbradiation gauge where $A_{0}=\partial^{i} A_{i}=0$. With $A_{0}=0$, from $J^{0}=0$, one obtains that $\dot{\theta}=0$ at the level of background. The $\nu=0$ component of the perturbed Maxwell equation (Eq. (9)) with the above gauge implies that $\delta \theta=0$. This is also consistent with the perturbed angular field equation (Eq. (7)) .

A non-zero spatial component of vector field at the background level may produce large anisotropies. In [44] the mechanism of magnetic seed production in the presence of an anisotropic gauge field was studied to some extent. It was shown that an anisotropic gauge field background will modify the inflation dynamics. It is found that the final produced magnetic field becomes highly suppressed as compared to the isotropic background. Also it is argued that a large anisotropic background may produce large anisotropies on CMB which may be detectable and need further studies. Here, in order to simplify the analysis and, in the light of [44], to have an optimum primordial magnetic field production, we assume that $\vec{A}=0$ at the background level. We need to check if this is a consistent solution of the background inflationary dynamics. To this end, we note that in our isotropic background, $F_{i j}=J^{i}=0$ and one can easily check that $\vec{A}=\dot{\vec{A}}=0$ is a consistent solution of Eqs. (7)-(9) and the Einstein equations. In other words, the $\rho$ field and the gauge field equations of motion decouple from each other. One also has to check that this ansatz is stable against perturbations. To see this, we note that the mass squared term created for the gauge field via spontaneous symmetry breaking (the Higgs mechanism) is positive, given by $e^{2} \rho^{2} / 2$, which indicates there is no tachyon in the mass spectra of the gauge field.

With this ansatz, at the background level, the independent equations of motion are

$$
\begin{aligned}
0 & =\ddot{\rho}+3 H \dot{\rho}+V_{\rho} \\
3 H^{2} M_{P}^{2} & =\frac{1}{2} \dot{\rho}^{2}+V(\rho) .
\end{aligned}
$$


The interesting result is that the inflation is completely driven by the radial field $\rho$ and the angular field $\theta$ is gauged by $U(1)$. As in conventional single field models of inflation, one can choose $V(\rho)$ to be flat enough to support an extended period of inflation. To be specific, below we consider the chaotic inflationary models. For potential $V=m^{2} \rho^{2} / 2$, the number of e-folding, $N_{e}$, is given by $4 M_{P}^{2} N_{e} \simeq \rho_{i}^{2}$, where $\rho_{i}$ is the initial value of the scalar field. To solve the flatness and horizon problem, we may take $N_{e}=60$, corresponding to $\rho_{i} \sim 10 M_{P}$. Furthermore, to fit the WMAP normalization for the density perturbation, $P_{\mathcal{R}} \sim 2.4 \times 10^{-9}$, one requires that $m \sim 6 \times 10^{-6} M_{P}$. For the quartic inflationary potential $V=\lambda \phi^{4} / 4$, to fit the the data one requires that $\lambda \simeq 10^{-13}$ and $\rho_{i} \sim 10 M_{P}$.

\section{MAGNETIC FIELD PRODUCTION DURING INFLATION}

Here we study magnetic field production during inflation. As is well-known, magnetic field production in FRW backgrounds is heavily suppressed due to the conformal invariance. To produce a significant amount of magnetic field, conformal invariance of the Maxwell equation should be broken. As we see from Eqs. (6) and (9), the conformal invariance is broken via spontaneous breaking of $U(1)$ symmetry where a mass term for the photon is generated dynamically through the coupling of $\rho$ field to $A_{\mu}$. The possibility of generating magnetic field during inflation due to $U(1)$ symmetry breaking by a complex field is studied extensively in literature [5, 11, 14, 16, 18]. In these models the complex scalar field is usually different than the inflaton field and it is assumed that the mechanism of magnetic field production happens on top of the inflationary background without affecting the dynamics of inflation. However, in our model we assume that the inflaton field is the same as the complex scalar field, i.e. the field $\rho$. This has the advantage that the parameters controlling the magnitude of magnetic fields production are directly related to the parameters that control the inflationary predictions, such as $N_{e}$ and density perturbations.

The vector perturbations are generated only quantum mechanically during inflation. To study their evolution and production, we go to Fourier space where the equation for the spatial components of the vector field is

$$
A_{i k}^{\prime \prime}+2 \frac{I^{\prime}}{I} A_{i k}^{\prime}+\left(k^{2}+\frac{e^{2}}{I^{2}} a^{2} \rho^{2}\right) A_{i k}=0,
$$

where $k$ is the comoving wave number. Hereafter, the prime indicates the derivative with 
respect to the conformal time $d \tau=d t / a(t)$. This equation represents a damped harmonic oscillator with a time-dependent mass. One can get rid of the first derivative term in Eq. (15) using $\vec{v}_{k}=I(\tau) \vec{A}_{k}$, so

$$
v_{k}^{\prime \prime}+\left(k^{2}-\frac{I^{\prime \prime}}{I}+\frac{e^{2}}{I^{2}} a^{2} \rho^{2}\right) v_{k}=0,
$$

For convenience we omits the indices $i$. As in [43] we consider the following ansatz for the gauge kinetic coupling

$$
I(\tau)=I_{f}\left(\frac{a}{a_{f}}\right)^{-p},
$$

where $I_{f}$ and $a_{f}$ are the values of $I(\tau)$ and the scale factor at the end of inflation, respectively. We expect that $I_{f} \lesssim 1$. Noting that the gauge kinetic coupling is inversely related to $I$, a negative value of $p$ corresponds to the case when gauge coupling is very large at the beginning of inflation and perturbative analysis is not reliable in the beginning of inflation. On the other hand, for a positive value of $p$ the gauge coupling is very small at the beginning of inflation and with the assumption $I_{f} \lesssim 1$ the perturbative gauge theory is under control for all time. For this purpose, we take $p \geq 0$ in our analysis below. Plugging the ansatz Eq. (17) into Eq. (16) yields

$$
v_{k}^{\prime \prime}+\left(k^{2}-\frac{p(p-1)}{\tau^{2}}+\frac{\beta}{\tau^{2 p+2}}\right) v_{k}=0,
$$

where

$$
\beta \equiv \frac{e^{2}\left\langle\rho^{2}\right\rangle}{I_{f}^{2} a_{f}^{2 p} H^{2 p+2}} .
$$

To obtain Eq. (18) the relation $a \simeq-1 / H \tau$ is used during the slow-roll inflation, where $H$ is the Hubble constant during inflation. Also we assume that $\rho$ is changing very slowly during inflation so we replace $\rho^{2}$ by its average value $\left\langle\rho^{2}\right\rangle$.

The amplitude of the magnetic field , $\delta_{B}$, at the end of inflation is given by [43]

$$
\delta_{B}\left(\tau_{f}\right)=\frac{k^{5 / 2}\left|v_{k}\left(\tau_{f}\right)\right|}{\sqrt{2} \pi a_{f}^{2} I_{f}},
$$

where $\left|v_{k}\left(\tau_{f}\right)\right|$ is the magnitude of $v_{k}$ at the end of inflation.

Eq. (18) can not be analytically solved for arbitrary value of $p$. Before considering general positive value of $p$, first we consider the particular case of $p=0$ corresponding to a constant gauge kinetic coupling, $I=1$. 


\section{A. Constant Gauge Kinetic Coupling, $p=0$}

For $p=0$ the solution of Eq. (18) are given in terms of Hankel functions

$$
\begin{aligned}
v_{k} & =A_{k} \\
& =\frac{\sqrt{\pi|\tau|}}{2} e^{i \pi\left(1+2 \nu_{0}\right) / 4}\left[b_{1} H_{\nu_{0}}^{(1)}(k|\tau|)+b_{2} H_{\nu_{0}}^{(2)}(k|\tau|)\right],
\end{aligned}
$$

where $\nu_{0}^{2}=1 / 4-\beta$. To match the initial vacuum state

$$
v_{k}=\frac{e^{-i k \tau}}{\sqrt{2 k}},
$$

one requires that $b_{1}=1$ and $b_{2}=0$ in Eq. (21). On the other hand, for long wavelength modes, $k|\tau| \rightarrow 0$, and by using the asymptotic relation

$$
H_{\nu_{0}}^{(1)}(k|\tau|) \rightarrow-\frac{i}{\pi} \Gamma\left(\nu_{0}\right)\left(\frac{k|\tau|}{2}\right)^{-\nu_{0}},
$$

at the end of inflation one obtains

$$
\delta_{B}\left(\tau_{f}\right) \simeq \frac{\Gamma\left(\nu_{0}\right) 2^{\nu_{0}-3 / 2}}{\pi^{3 / 2} I_{f}} H^{2}\left(\frac{a_{f} H}{k}\right)^{\nu_{0}-5 / 2},
$$

where $\Gamma(x)$ is the Gamma function. Noting that the real value of $\nu_{0}$ can not exceed 1/4 the amplitude of magnetic field in Eq. (24) is hugely scale suppressed.

To calculate the amplitude of magnetic fields after inflation we should take into account the expansion of the Universe which dilutes the magnetic field energy density, $B_{i} B^{i}$, like radiation and $\delta_{B}$ decreases like $a^{-2}$. Assuming that the preheating and reheating happens instantly, followed by a radiation-dominated Universe, one obtains that $a_{d e c} / a_{f} \simeq \sqrt{H M_{P}} / T_{d e c}$ where $T_{d e c}$ and $a_{d e c}$ are the values of the temperature and scale factor at the time of decoupling, $t_{d e c}$, respectively. For $H \simeq 10^{-6} M_{P}$ one obtains $a_{f} \sim 10^{-29}$ and $a_{d e c} / a_{f} \simeq 10^{26}$ where we have set the magnitude of the scale factor today equal to unity, $a_{0}=1$. Putting all together, with $H^{2} \simeq 10^{-12} M_{P}^{2} \sim 10^{46}$ Gauss, the amplitude of the magnetic fields at the time of decoupling is $\delta_{B}\left(t_{d e c}\right) \simeq 10^{-6} \times 10^{11\left(2 \nu_{0}-5\right)} \lesssim 10^{-50}$ Gauss on comoving scales $k^{-1}=1 M p c \sim 10^{38} \mathrm{GeV}^{-1}$. This seed magnetic field is too small to be amplified by galactic dynamo mechanism into the current observed values.

One may compare the case $p=0$ here to the models studied in [5, 16, 43] where the gauge field is coupled to the Ricci scalar via the mass term $\beta R A_{\mu} A^{\mu} / 2$ and the gauge invariance and the conformal invariance are broken explicitly. However, in our model the 
gauge invariance and the conformal invariance are broken only dynamically (spontaneously) when the inflaton field is dislocated from the minimum during inflation. In their cases the conformal coupling parameter $\beta$ can have a negative value so a negative mass squared can be generated for the gauge field. This results in an amplification of the magnetic field, and one has to check that its strong back reactions do not destroy the background inflationary setup [43]. However, in our case $\beta>0$ and a positive mass squared term is created for the gauge field and the magnetic field is more suppressed compared to the case of a massless gauge field where $\beta=0$ and $\nu=1 / 2$.

\section{B. Magnetic Fields from Time Varying Gauge Kinetic Coupling, $p>0$}

To keep the gauge kinetic coupling under perturbative control, we take $p>0$. As it is obvious, Eq. (18) can not be solved analytically, so we rely on the numerical solution and asymptotic behaviors.

During early stage of inflation the first two terms inside the bracket of Eq. (18) dominate. The last term dominates only at the final stage of inflation when $\tau \rightarrow 0$. For the last term to dominate over the second term one requires that

$$
\Omega \equiv \beta\left|\tau_{f}\right|^{-2 p}=\frac{e^{2}\left\langle\rho^{2}\right\rangle}{I_{f}^{2} H^{2}} \gg 1 .
$$

To have an estimate of $\Omega$, consider the inflationary potential $V=m^{2} \rho^{2} / 2$. Using the relation $6 H^{2} M_{P}^{2}=m^{2} \rho^{2}$, one obtains

$$
\Omega=6\left(\frac{e M_{P}}{m I_{f}}\right)^{2} .
$$

To fit the COBE normalization of density perturbation, $m \sim 10^{-6} M_{P}$ so, for $e \sim I_{f} \sim 1$, $\Omega$ can be as large as $10^{12}$. Increasing(decreasing) the ratio $e / I\left(\tau_{f}\right)$ the magnitude of $\Omega$ increases(decreases). On the other hand, for $\lambda \phi^{4} / 4$ inflationary model, one obtains

$$
\Omega=\frac{12}{\lambda}\left(\frac{e M_{P}}{I_{f} \rho}\right)^{2} .
$$

For $\lambda \simeq 10^{-13}$ and $\rho \simeq 10 M_{P}, \Omega$ can be as large as $10^{12}$ like in the previous example.

In the early stage of inflation when the term containing $\beta$ is negligible in Eq. (18), the asymptotic solution is

$$
v_{k}^{\text {early }}(\tau)=\frac{\sqrt{\pi|\tau|}}{2} e^{i(1+2 \nu) \pi / 4} H_{\nu}^{(1)}(k|\tau|)
$$


where $\nu \equiv p-1 / 2$. At the final stage of inflation, the term containing $\beta$ dominates and the asymptotic solution of Eq. (18) is given by:

$$
v_{k}^{\text {final }}(\tau)=\frac{i \sqrt{\pi|\tau|}}{2}\left[c_{1} H_{\mu}^{(1)}\left(\frac{\sqrt{\beta}|\tau|^{-p}}{p}\right)+c_{2} H_{\mu}^{(2)}\left(\frac{\sqrt{\beta}|\tau|^{-p}}{p}\right)\right],
$$

where $\mu=1 / 2 p$ and $c_{1}$ and $c_{2}$ are the Bogoliubov coefficients. One needs to match solutions $v_{k}^{\text {early }}$ and $v_{k}^{\text {final }}$ at the transition time, $\tau_{c}$, where

$$
\beta\left|\tau_{c}\right|^{-2 p}=|p(p-1)|
$$

Since $\tau_{c}$ is expected to be close to the end of inflation, then $k \tau_{c} \rightarrow 0$ and $\tau_{c}$ given above is independent of $k$.

Demanding that both $v_{k}$ and $v_{k}^{\prime}$ to be continuous at $\tau_{c}$ fixes the coefficients $c_{1}$ and $c_{2}$

$$
c_{1,2}=\frac{ \pm \pi}{4 p} e^{i(1+2 \nu) \pi / 4}\left[\sqrt{\beta}\left|\tau_{c}\right|^{-p} H_{\nu}^{(1)} H_{\mu}^{(2,1)^{\prime}}+k\left|\tau_{c}\right| H_{\nu}^{(1)^{\prime}} H_{\mu}^{(2,1)}\right]
$$

where the arguments of $H_{\mu}$ are $\sqrt{\beta}\left|\tau_{c}\right|^{-p} / p$ while that of $H_{\nu}$ are $\left|k \tau_{c}\right|$.

It is instructive to introduce the dimensionless variable $x \equiv k|\tau|$, and rewrite Eq. (30) as

$$
x_{c}=\left[\frac{\Omega}{|p(p-1)|}\right]^{1 / 2 p} x_{f}
$$

where $x_{f}=k\left|\tau_{f}\right|$.

Figure 1 shows the value of $x_{c} / x_{f}$ as a function of $p$. For a given value of $p$, by increasing $\Omega$ the value of $x_{c}$ goes to the larger values as expected. We note that with $k^{-1}=1 M p c$ and $H \simeq 10^{-6} M_{P}$ one has $x_{f} \simeq 10^{-22}$. This indicates that $x_{c} \rightarrow 0$ as verified from Eq. (30) and also from Figure 1. Physically, this means that the last term in Eq. (18) dominates at the late stages of inflation.

The full Numerical solution of Eq. (18) for typical value of $p=2.3$ and $\Omega=10^{12}$ along with the asymptotic behaviors at the initial stage, Eq. (28), and the asymptotic solution at the final stage, Eq. (29), are plotted in Figure 2. One can check that the approximate solution Eq. (29) is in a good agreement with the full numerical solution. The transition point, $x_{c}$, where the two solutions Eq. (29) and (28) are matched smoothly together can be seen in Figure 2.

As explained above, we expect that $k \tau_{c} \rightarrow 0$, which can be used to simplify the value of $c_{1}$ and $c_{2}$. Using the asymptotic form Eq. (23), one obtains that

$$
\left|c_{1}\right| \sim\left|c_{2}\right| \sim\left|k \tau_{c}\right|^{-\nu}
$$




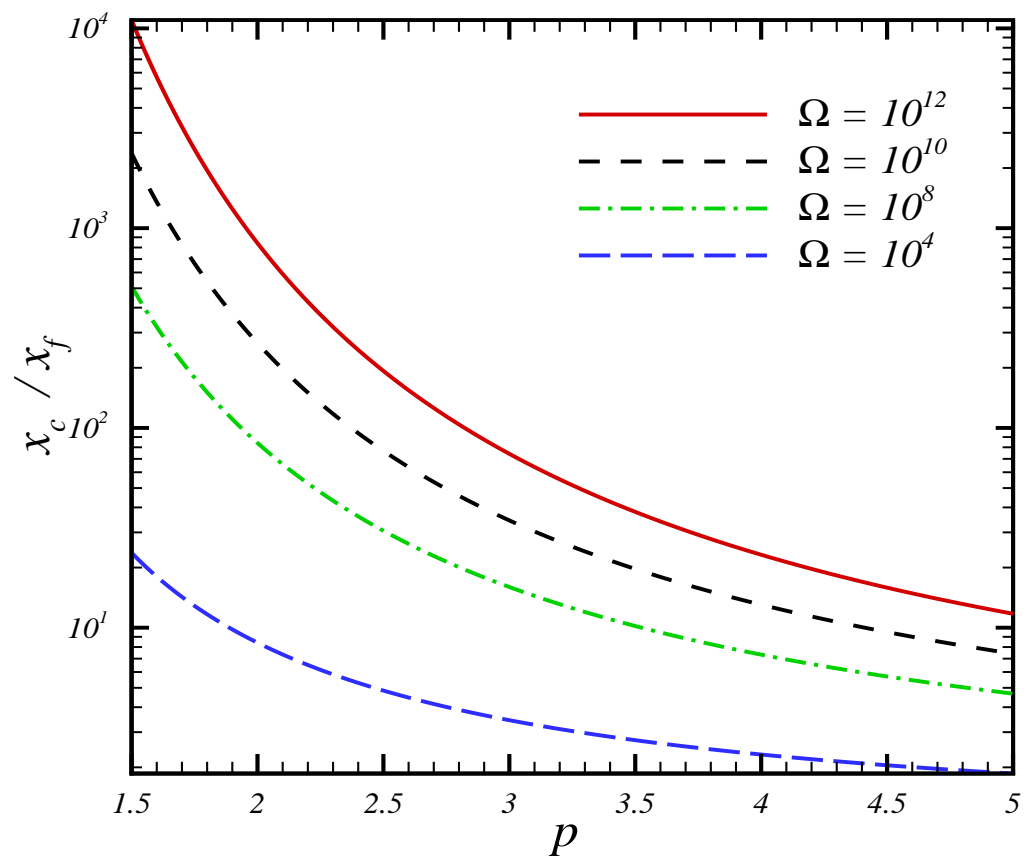

FIG. 1: Here we plot the behavior of $x_{c} \equiv k\left|\tau_{c}\right|$ versus $p$ given by Eq. (32) for various values of $\Omega$. We took $x_{f}=10^{-22}$.

and

$$
\left|v_{k}^{\text {final }}\left(\tau_{f}\right)\right| \sim \sqrt{\left|\tau_{f}\right|} \Omega^{-\frac{1}{4}}\left|k \tau_{c}\right|^{-\nu}
$$

where to get the final answer use was made of the large argument limit of the Hankel functions $H_{\mu}(x) \sim x^{-1 / 2}$ for $x \gg 1$. One the other hand, using Eq. (30) one can express $\tau_{c}$ in favor of $\tau_{f}$ via $\tau_{c} / \tau_{f} \simeq \Omega^{1 / 2 p}$. Putting all together, and noting that $\nu=p-1 / 2$ and neglecting factors of order unity one has

$$
\left|v_{k}^{\text {final }}\left(\tau_{f}\right)\right| \sim \sqrt{\left|\tau_{f}\right|} \Omega^{-\frac{3}{4}+\frac{1}{4 p}}\left|k \tau_{f}\right|^{-\nu}
$$

which from Eq. (20) results in

$$
\delta_{B}\left(\tau_{f}\right) \sim \Omega^{-\frac{3}{4}+\frac{1}{4 p}} H^{2}\left(\frac{a_{f} H}{k}\right)^{p-3} .
$$

As expected, up to term containing the factor of $\Omega$, this is the same as in [43]. Since $\Omega \gg 1$, one may expect that our value of $\delta_{B}\left(\tau_{d e c}\right)$ would be more suppressed compared to the result of [43]. On the other hand, due to the presence of $\Omega$, the allowed range of $p$ 


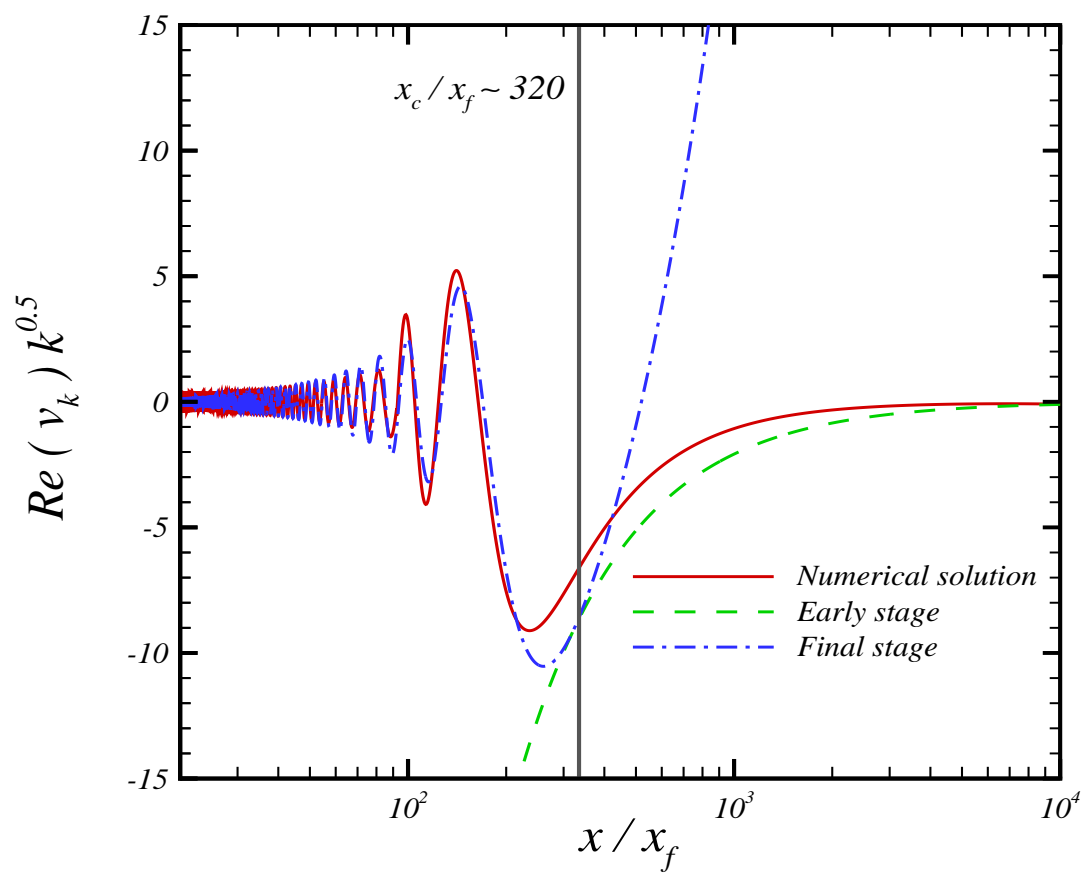

FIG. 2: The numerical solution of the real part of Eq. (18) as a function of $x=k|\tau|$. To make the presentation more transparent, the vertical axis is scaled down by a factor of $10^{-25}$. The solid (red) curve indicates the full numerical solution whereas the other two curves indicate the early and the late time solutions, given by Eqs. (28) and (29). We took $p=2.3, \Omega=10^{12}$ and $x_{f}=10^{-22}$. The transition point, $x_{c}$, calculated from Eq. (30), is indicated by the vertical line. We see that at $x_{c}$ the solutions (28) and (29) match smoothly and are in a good agreement with the full numerical solution.

increases as compared to that in [43]. One has to check whether the increase in range of $p$ can compensate the suppression from large $\Omega$ effect. In the examples below we will show that unfortunately Eq. (36) gives smaller value for the magnetic fields than those obtained in [43] in the absence of $\Omega$.

As in [43], there is an upper bound on $p$, determined by the requirement that the energy density from the vector field, $\epsilon_{E M}$, does not exceed the background inflationary energy density. This is equivalent to $\epsilon_{E M}<M_{P}^{2} H^{2}$. There are two sources for $\epsilon_{E M}$ coming from Eq. (11). The first one, which was also considered in [43], is $\epsilon_{E M}^{(F)} \equiv-\frac{1}{4} I(t)^{2} F_{\alpha \beta} F^{\alpha \beta}+I(t)^{2} F_{0 \alpha} F_{0}^{\alpha}$ and the second one is the contribution from the gauge coupling $e$ in the form of $\epsilon_{E M}^{(e)} \equiv$ $J_{\alpha} J^{\alpha} / \rho^{2}|g|=e^{2} \rho^{2} A_{i} A^{i} / 2$. Below we show that $\epsilon_{E M}^{(e)}$ dominates over $\epsilon_{E M}^{(F)}$ by a factor of $\Omega$. 
Following [43], one obtains

$$
\begin{aligned}
\epsilon_{E M}^{(e)}\left(\tau_{f}\right) & =\frac{1}{2} e^{2} \rho^{2}\left\langle 0\left|A_{i} A^{i}\right| 0\right\rangle \\
& \sim \frac{\Omega H^{2}}{4 \pi^{2} a_{f}^{2}} \int_{a_{i} H}^{a_{f} H} d k k^{2}\left|v_{k}\left(\tau_{f}\right)\right|^{2},
\end{aligned}
$$

where $a_{i}$ is the initial size of the scale factor at the start of inflation. Using Eq. (34), one obtains

$$
\epsilon_{E M}^{(e)}\left(\tau_{f}\right) \sim \Omega^{-\frac{1}{2}+\frac{1}{2 p}} \frac{H^{4}}{4 \pi^{2}} \times \begin{cases}\frac{1}{2-p} & p<2 \\ \ln \left(\frac{a_{f}}{a_{i}}\right) & p=2 \\ \frac{1}{p-2}\left(\frac{a_{f}}{a_{i}}\right)^{2(p-2)} & p>2\end{cases}
$$

where to get the final result, the relation $\tau_{f} \simeq-1 / a_{f} H$ is used during slow-roll inflation.

On the other hand, $\epsilon_{E M}^{(F)}$ is given by [43] $\epsilon_{E M}^{(F)} \sim a_{f}^{-4} \int_{a_{i} H}^{a_{f} H} d k k^{2}\left|v_{k}^{\prime}\left(\tau_{f}\right)\right|^{2}$. One can show that $\epsilon_{E M}^{(F)} \simeq \Omega^{-1} \epsilon_{E M}^{(e)}$ and with $\Omega \gg 1$, the requirement $\epsilon_{E M}^{(e)}<M_{P}^{2} H^{2}$ is the stronger constraint that has to be satisfied.

From Eq. (36), to have a large enough magnetic field, it is optimum to have $p>2$. However, the magnitude of $p$ is controlled by the value of $\Omega$ and $a_{f} / a_{i}$. For example, with $H \sim 10^{-6} M_{P}, N_{e}=\ln \left(a_{f} / a_{i}\right)=60$ and $\Omega=10^{12}$ which is naturally obtained in chaotic inflationary models, the constraint $\epsilon_{E M}^{(e)}<M_{P}^{2} H^{2}$ results in $p \lesssim 2.3$. This results in $\delta_{B}\left(t_{\text {dec }}\right) \sim 10^{-29}$ Gauss on $k^{-1} \sim$ Mpc scales. This value of magnetic seed may be marginally acceptable in the light of [4]. Reducing $\Omega$ results in an increase in $\delta_{B}\left(t_{d e c}\right)$. For example, choosing $\Omega=10^{4}$, one obtains $\delta_{B}\left(t_{d e c}\right) \sim 10^{-25}$ Gauss. With $I_{f} \sim 1$, this value of $\Omega$ corresponds to $e \sim 10^{-4}$ which indicates a severe fine-tuning. On the other hand, in the absence of $\Omega$ factor, one obtains $p \lesssim 2.2$ and $\delta_{B}\left(t_{d e c}\right) \sim 10^{-23}$ Gauss.

\section{CONCLUSION}

We considered a model where the inflaton field is a charged scalar field coupled to a $U(1)$ gauge field with a time-varying gauge kinetic coupling. The gauge field is frozen in the classical background and is excited quantum mechanically. The amplitudes of magnetic fields produced down to the end of inflation and at the time of decoupling are calculated. Our model has features in common with models such as in [5, 11, 14, 16, 18] where a charged scalar field is present in an inflationary background. However, since in our model the charged 
scalar field itself is the inflaton field, the prediction of magnetic fields are directly controlled by the inflationary parameters such as $e, N_{e}$ and $m$ in $m^{2} \rho^{2}$ or $\lambda$ in $\lambda \phi^{4}$ models. As in [43], the main constraint controlling the magnitude of $\delta_{B}$ comes from the requirement that the back reaction from the gauge field does not destroy the inflationary background. This in turn imposes an upper bound on the range of parameter $p$. Our model predicts values of $\delta_{B}\left(t_{d e c}\right)$ somewhat smaller than what is obtained in [43] in the absence of charged scalar field. However, our model predicts a higher value of spectral index parameter, $p$, for the primordial magnetic field. With natural parameter values, our model predicts that magnetic field at the order of $\delta_{B}\left(t_{d e c}\right) \sim 10^{-29}$ Gauss can be created on $k^{-1} \sim$ Mpc scales. This value may be marginally acceptable in the case of very efficient dynamo mechanism.

In this model important issues such as the amplification of magnetic fields during preheating and subsequent suppressions via electric conductance are not considered. Specially, since the gauge field obtains a time-dependent mass, as is evident in Eq. (15), the effects from parametric resonance and significant amplification of magnetic field during preheating stage may play some important roles [13, 14, 16]. This may help to relax the bounds above by few orders of magnitude.

In this work we have turned off the background gauge field classically. Although this is a consistent solution, but it is not the most natural solution and requires an additional fine-tuning in our model. In a future work [46] we study the case in our model where the background gauge field is not turned off classically. For this, one has to search for the parameter space where the gauge-field energy density does not dominate over the inflaton field energy density such that the produced anisotropies are within the observational bounds

from CMB. This is similar to the analysis of [47] except that in their model there is no gauge coupling, $e=0$, and $\beta=0$.

\section{ACKNOWLEDGEMENT}

We would like to thank Bruce Bassett, Robert Brandenberger, Jim Cline, Paolo Creminelli, Massimo Giovannini, Lev Kofman and Shahin Sheikh-Jabbari for valuable discussions and comments. We also thank the anonymous PRD referee for the insightful critical comments and for useful suggestions on the draft. H.F. would like to thank Perimeter Institute and McGill University for hospitality where this work was completed. 


\section{References}

[1] D. Grasso and H. R. Rubinstein, "Magnetic fields in the early universe," Phys. Rept. 348, 163 (2001) arXiv:astro-ph/0009061.

[2] L. M. Widrow, "Origin of Galactic and Extragalactic Magnetic Fields," Rev. Mod. Phys. 74, 775 (2002) arXiv:astro-ph/0207240].

[3] M. Giovannini, "Primordial magnetic fields," arXiv:hep-ph/0208152,

M. Giovannini, "Magnetic fields, strings and cosmology," Lect. Notes Phys. 737, 863 (2008) arXiv:astro-ph/0612378].

[4] A. C. Davis, M. Lilley and O. Tornkvist, "Relaxing the Bounds on Primordial Magnetic Seed Fields," Phys. Rev. D 60, 021301 (1999) arXiv:astro-ph/9904022.

[5] M. S. Turner and L. M. Widrow, "Inflation Produced, Large Scale Magnetic Fields," Phys. Rev. D 37, 2743 (1988).

[6] B. Ratra, "Cosmological 'seed' magnetic field from inflation," Astrophys. J. 391, L1 (1992).

[7] A. Dolgov, "Breaking Of Conformal Invariance And Electromagnetic Field Generation In The Universe," Phys. Rev. D 48, 2499 (1993) arXiv:hep-ph/9301280.

[8] D. Lemoine and M. Lemoine, "Primordial magnetic fields in string cosmology," Phys. Rev. D 52, 1955 (1995).

[9] M. Gasperini, M. Giovannini and G. Veneziano, "Primordial magnetic fields from string cosmology," Phys. Rev. Lett. 75, 3796 (1995) arXiv:hep-th/9504083.

[10] E. A. Calzetta, A. Kandus and F. D. Mazzitelli, "Primordial magnetic fields induced by cosmological particle creation," Phys. Rev. D 57, 7139 (1998) arXiv:astro-ph/9707220];

E. A. Calzetta and A. Kandus, "Self consistent estimates of magnetic fields from reheating," Phys. Rev. D 65, 063004 (2002) arXiv:astro-ph/0110341].

[11] F. Finelli and A. Gruppuso, "Resonant amplification of gauge fields in expanding universe," Phys. Lett. B 502, 216 (2001) arXiv:hep-ph/0001231.

[12] M. Giovannini and M. E. Shaposhnikov, "Primordial magnetic fields from inflation?," Phys. Rev. D 62, 103512 (2000) arXiv:hep-ph/0004269.

[13] M. Giovannini and M. E. Shaposhnikov, "Primordial magnetic fields from inflation?," 
arXiv:hep-ph/0011105.

[14] A. C. Davis, K. Dimopoulos, T. Prokopec and O. Tornkvist, "Primordial spectrum of gauge fields from inflation," Phys. Lett. B 501, 165 (2001) [Phys. Rev. Focus 10, STORY9 (2002)] arXiv:astro-ph/0007214].

[15] M. Gasperini, "A new mechanism for the generation of primordial seeds for the cosmic magnetic fields," Phys. Rev. D 63, 047301 (2001) arXiv:astro-ph/0009476.

[16] B. A. Bassett, G. Pollifrone, S. Tsujikawa and F. Viniegra, "Preheating as cosmic magnetic dynamo," Phys. Rev. D 63, 103515 (2001) arXiv:astro-ph/0010628].

[17] A. Ashoorioon and R. B. Mann, "Generation of cosmological seed magnetic fields from inflation with cutoff," Phys. Rev. D 71, 103509 (2005) arXiv:gr-qc/0410053.

[18] M. Giovannini, "On the variation of the gauge couplings during inflation," Phys. Rev. D 64, 061301 (2001) arXiv:astro-ph/0104290.

[19] K. Bamba and J. Yokoyama, "Large-scale magnetic fields from inflation in dilaton electromagnetism," Phys. Rev. D 69, 043507 (2004) arXiv:astro-ph/0310824.

[20] K. Bamba, C. Q. Geng and S. H. Ho, "Large-scale magnetic fields from inflation due to ChernSimons-like effective interaction," JCAP 0811, 013 (2008) arXiv:0806.1856 [astro-ph]];

K. Bamba, N. Ohta and S. Tsujikawa, "Generic estimates for magnetic fields generated during inflation including Dirac-Born-Infeld theories," Phys. Rev. D 78, 043524 (2008) arXiv:0805.3862 [astro-ph]];

K. Bamba, "The interrelation between the generation of large-scale electric fields and that of large-scale magnetic fields during inflation," JCAP 0710, 015 (2007) arXiv:0710.1906 [astro$\mathrm{ph}]$;

K. Bamba, "Property of the spectrum of large-scale magnetic fields from inflation," Phys. Rev. D 75, 083516 (2007) arXiv:astro-ph/0703647].

[21] M. A. Ganjali, "DBI with primordial magnetic field in the sky," JHEP 0509, 004 (2005) arXiv:hep-th/0509032.

[22] K. Bamba and M. Sasaki, "Large-scale magnetic fields in the inflationary universe," JCAP 0702, 030 (2007) arXiv:astro-ph/0611701.

[23] M. Giovannini, "Magnetogenesis, spectator fields and CMB signatures," Phys. Lett. B 659, 661 (2008) arXiv:0711.3273 [astro-ph]].

[24] K. Bamba and J. Yokoyama, "Large-scale magnetic fields from dilaton inflation in noncom- 
mutative spacetime," Phys. Rev. D 70 (2004) 083508.

[25] O. Bertolami and R. Monteiro, "Varying electromagnetic coupling and primordial magnetic fields," Phys. Rev. D 71 (2005) 123525.

[26] J. M. Salim, N. Souza, S. E. Perez Bergliaffa and T. Prokopec, "Creation of cosmological magnetic fields in a bouncing cosmology," JCAP 0704 (2007) 011.

[27] J. Martin and J. Yokoyama, "Generation of Large-Scale Magnetic Fields in Single-Field Inflation," JCAP 0801 (2008) 025.

[28] K. Bamba and S. D. Odintsov, "Inflation and late-time cosmic acceleration in non-minimal Maxwell- $F(R)$ gravity and the generation of large-scale magnetic fields," JCAP 0804 (2008) 024 .

[29] K. Bamba, C. Q. Geng and S. H. Ho, "Large-scale magnetic fields from inflation due to Chern-Simons-like effective interaction," JCAP 0811 (2008) 013.

[30] W. D. Garretson, G. B. Field and S. M. Carroll, "Primordial magnetic fields from pseudoGoldstone bosons," Phys. Rev. D 46 (1992) 5346.

[31] F. D. Mazzitelli and F. M. Spedalieri, "Scalar electrodynamics and primordial magnetic fields," Phys. Rev. D 52 (1995) 6694.

[32] M. Novello, L. A. R. Oliveira and J. M. Salim, "Direct Electrogravitational Couplings And The Behavior Of Primordial Large Scale Magnetic Fields," Class. Quant. Grav. 13 (1996) 1089.

[33] O. Bertolami and D. F. Mota, "Primordial magnetic fields via spontaneous breaking of Lorentz invariance," Phys. Lett. B 455 (1999) 96.

[34] M. Giovannini, "Magnetogenesis and the dynamics of internal dimensions," Phys. Rev. D 62 (2000) 123505.

[35] K. Dimopoulos, T. Prokopec, O. Tornkvist and A. C. Davis, "Natural magnetogenesis from inflation," Phys. Rev. D 65 (2002) 063505.

[36] T. Prokopec and E. Puchwein, "Photon mass generation during inflation: de Sitter invariant case," JCAP 0404 (2004) 007. T. Prokopec and E. Puchwein, "Nearly minimal magnetogenesis," Phys. Rev. D 70 (2004) 043004.

[37] K. Enqvist, A. Jokinen and A. Mazumdar, "Seed perturbations for primordial magnetic fields from MSSM flat directions," JCAP 0411 (2004) 001.

[38] M. R. Garousi, M. Sami and S. Tsujikawa, "Generation of electromagnetic fields in string 
cosmology with a massive scalar field on the anti D-brane," Phys. Lett. B 606 (2005) 1.

[39] J. E. Madriz Aguilar and M. Bellini, "Stochastic gravitoelectromagnetic inflation," Phys. Lett. B 642 (2006) 302.

[40] F. Agustin Membiela and M. Bellini, "Power spectrum of large-scale magnetic fields from Gravitoelectromagnetic inflation with a decaying cosmological parameter," arXiv: 0712.3032 [hep-th].

[41] L. Campanelli, P. Cea, G. L. Fogli and L. Tedesco, "Inflation-Produced Magnetic Fields in Nonlinear Electrodynamics," Phys. Rev. D 77 (2008) 043001; L. Campanelli, P. Cea, G. L. Fogli and L. Tedesco, "Inflation-Produced Magnetic Fields in $R^{n} F^{2}$ and $I F^{2}$ models," Phys. Rev. D 77 (2008) 123002.

[42] L. Campanelli, "Helical Magnetic Fields from Inflation," Int. J. Mod. Phys. D 18, 1395 (2009) arXiv:0805.0575 [astro-ph]].

[43] V. Demozzi, V. Mukhanov and H. Rubinstein, "Magnetic fields from inflation?," arXiv:0907.1030 [astro-ph.CO].

[44] S. Kanno, J. Soda and M. a. Watanabe, "Cosmological Magnetic Fields from Inflation and Backreaction," arXiv:0908.3509 [astro-ph.CO].

[45] A. Dolgov and J. Silk, "Electric charge asymmetry of the universe and magnetic field generation," Phys. Rev. D 47, 3144 (1993).

[46] R. Emami, H. Firouzjahi, S. Movahed and M. Zarei, work in progress .

[47] M. a. Watanabe, S. Kanno and J. Soda, "Inflationary Universe with Anisotropic Hair," Phys. Rev. Lett. 102, 191302 (2009) [arXiv:0902.2833 [hep-th]]. 